\title{
Anomalous diffusion on dynamical networks: A model for interacting epithelial cell migration
}

\author{
Stefan Thurner ${ }^{a, b \uparrow \uparrow \uparrow, ~ N i k o l a u s ~}$ Wick $^{c, d}$, Rudolf \\ Hanel $^{e}$, Roland Sedivy ${ }^{c}$, and Lukas Huber ${ }^{d, f}$ \\ a Klinik für HNO, Universität Wien; Austria, \\ ${ }^{b}$ Institut für Mathematik, NuHAG, Universität Wien; Austria, \\ c Klinisches Institut für Pathologie, Universität Wien; Austria, \\ ${ }^{d}$ Institute for Molecular Pathology, Vienna; Austria, \\ e Institut für Biomedizinische Technik und Physik, Universität Wien; Austria, \\ $f$ Institut für Anatomie und Histologie, Universität Innsbruck; Austria
}

\begin{abstract}
We propose a model for cell migration where epithelial cells are able to detect trajectories of other cells and try to follow them. As cells move along in 2D cell culture, they mark their paths by loosing tiny parts of cytoplasm. Any cell moving on a surface where other cells have moved before faces a network of cell trajectories, which it tries to restrict its motion onto. With the Tsallis modification of classical thermodynamics one can solve the relevant Fokker-Planck like equation and obtain experimentally testable distribution functions. We compare the model to experimental data of normal mammary epithelial cells and cells which have been genetically manipulated to change their degree of cell-cell interaction.
\end{abstract}

PACS: 87.17.Jj, 05.40.-a,05.45.Tp 87.17.Aa,

Keywords: anomalous diffusion, Tsallis distributions, cell migration

ศศ Correspondence should be addressed to:

Prof. Stefan Thurner, PhD, PhD; HNO, AKH-Wien, University of Vienna

Währinger Gürtel 18-20; A-1090 Vienna, Austria

Tel.: ++43140400 2099; Fax: ++431404003332

e-mail: thurner@univie.ac.at 


\section{INTRODUCTION}

A generalization of classical Boltzmann thermodynamics as recently introduced by Tsallis 11, 2, 3] has offered a beautiful way of describing the statistics of a multitude of physical systems, which generally involve some sort of long-range interactions, ranging from astrophysics [4, 5] to low dimensional dissipative chaotic systems [6]. Systems described by this thermodynamics are often referred to as "non-extensive". This same modification of classical thermodynamics also seems to offer a natural understanding of the subject of anomalous diffusion. Anomalous diffusion can be thought of the propagation of particles which are restricted to move on a certain subspace of full two or three space, such as for example the propagation of water molecules in a sponge, or the flow of crude oil in porous soil. In this work we argue that the motion of living and interacting epithelial cells can be seen as a variant of anomalous diffusion on a network which changes over time. We support the idea by experimental evidence. Our experimental findings moreover strongly support very recent work on movements of interacting Hydra cells [7].

A living epithelial cell e.g. in two-dimensional cell culture is not a static object but moves continuously, a process which is called cellular migration [8, 9]. Cell migration is of vital importance in physiological processes such as wound healing or understanding cancerogenesis. For example, intact mammary gland tissue is stable during phases of milk secretion. In residual phases of female estrous cycle, however, it is subject to structural remodeling where cells become dynamic entities. They proliferate and migrate, eventually forming new functional secretory units [10, 11, 12]. Dysregulation of these events is believed to contribute to cancerogenesis 13, 14, 15]. In-vitro experiments preserve important aspects of this epithelial biology [16, 17], and migration of single cells has been studied in cell culture systems on molecular and mechanical levels $8,9,18$.

Single cells are by no means passive movers and governed by chance, but influence and control their movements themselves. For these movements to occur morphological changes are necessary which are realized in the formation of distinct subcellular domains. In Fig. 1a a single cell is shown during movement. After the formation of a so-called lamellipodium, (a V-shaped protrusion) this part of the cell attaches to the cell culture surface. The cell body is then pulled towards the front part ("leading edge") via the activation of mechanical intracellular linkages. Eventually the "trailing" edge, a thin, elongated structure at the 
opposite side of the cell with respect to the lamellipodium, detaches from the surface which allows a net translocation of the cell. In this "polarized" fashion the cell apparently crawls on the surface of the cell culture substrate, continuously readjusting its direction. During movement cells loose tiny parts of their cytoplasm, marking at least parts of their trajectories. It is known that cells change their movement patterns when the activity of proteins involved in cell adhesion is regulated up or down ward. When these proteins for example are suppressed, cells loose their ability to migrate in a polarized fashion [19, 20]. As several recent works on velocity distributions of cells show, it seems very likely that cell movement depends strongly on the surrounding of individual cells. Experimental results range from normal diffusion [21] to exponential velocity distributions [22]. It is the aim of this paper to show that an anomalous diffusion approach, already discussed in [7], might be general enough to explain the different experimental results on a common theoretical ground.

\section{THEORY}

To describe the dynamics of a single cell which has the ability to detect the presence of trajectories of other cells, we propose the following model. Imagine each cells paints its trail onto the surface. The paint slowly fades, and can not be seen after a certain time. Another cell which detects the line of paint tries to follow it as good as possible. From time to time the cell will be distracted from strict path following by random ("thermal") events. At the same time this cell also marks its trajectory with paint. As many cells move across the same surface there will evolve a network of paths. Since paint fades and new trajectories are added to the network, the network will undergo slow dynamical changes. Any cell equipped with a trail following mode will try to restrict its movements onto the network of trails of former cells. The situation here is much alike diffusion in porous media where particle trajectories are restricted onto certain pathways, and can not propagate through full twoor three-dimensional space. In the case of cell migration an individual cell tries to follow the dynamical "web" of trajectories other cells have taken before.

Modeling cell migration as a stochastic diffusion process of this kind clearly involves non-linearities in the associated Fokker-Planck like equations

$$
\frac{\partial}{\partial t} p(x, t)=-\frac{\partial}{\partial x}(F[p](x, t) p(x, t))+\frac{1}{2} \frac{\partial^{2}}{\partial x^{2}}(D[p](x, t) p(x, t))
$$


To incorporate the idea that cells follow the gradient of metabolites emitted by other cells along their trajectories, a non-linear flow-term of the form $F[p]=\frac{\partial}{\partial x} p$ would be reasonable. However, we neglect the flow-term in the following, since a posteriori we find almost no experimental evidence for it. To incorporate the anomalous diffusion idea, we decide the functional dependence of the diffusion parameter to be $D[p] p=p^{\nu}$. With this choice equation (四) can be solved by following the entropy approach by Tsallis [1], 3]. Here the the classical entropy definition $S=-\int p(u) \ln p(u) d u$ is modified to

$$
S_{q}=\frac{1-\int p(u)^{q} d u}{q-1} .
$$

By maximizing $S_{q}$ while keeping energy fixed it can be shown that the resulting $p(u)$, i.e.

$$
p_{q}(x, t)=\frac{\left(1-\beta(t)(1-q)(x-\langle x\rangle(t))^{2}\right)^{\frac{1}{1-q}}}{Z_{q}(t)}
$$

also solves Eq. (1) without the flow-term [2, 23]. Here $Z_{q}(t)$ is the generalized $q$ partition function. The relation of the Tsallis entropy factor $q$, and $\nu$ in Eq. (1) is given by $q=2-\nu$ [2]. Note that this distribution is a power law, and only in the limit $q \longrightarrow 1$ the classical Gaussian $p_{1}(x, t)=\frac{\exp ^{-\beta(t)(x-\langle x\rangle(t))^{2}}}{Z_{1}(t)}$ is recovered. It is relatively straight forward to derive the corresponding velocity distributions of the associated particles

$$
p(v)=B_{q}\left[1-(1-q) \frac{\beta m v^{2}}{2}\right]^{\frac{1}{1-q}}
$$

which has been derived for the first time in [24], and which reduces to the usual MaxwellBoltzmann result $p(v)=\left(\frac{m}{2 \pi k T}\right)^{\frac{3}{2}} \exp ^{-\frac{\beta m v^{2}}{2}}$ in the limit $q \longrightarrow 1$. Here $\frac{\beta m}{2}$ can be seen as an inverse mobility factor, meaning that large values of $\frac{\beta m}{2}$ are associated with small average cell velocities. If cell migration is in fact describable as anomalous diffusion governed by a Fokker-Planck equation like Eq. (1) with the special choice of $D[p]$, the cell velocity distribution function should follow the form of Eq. (四).

To further characterize the nature of the diffusion process we will also look at normalized temporal autocorrelation functions $A C(\tau)=\frac{1}{\sigma^{2}(\xi)}\langle\xi(t) \xi(t-\tau)\rangle_{t}$, and at two-point correlation functions $C(\tau)=\left\langle[\xi(t+\tau)-\xi(t)]^{2}\right\rangle_{t}$, of an underlying process $\xi(t)$, where $\langle.\rangle_{t}$ denotes the average over time $t$. For (fractional) Brownian motion, the correlator scales as $C(\tau) \sim \tau^{2 H}$, where $H$ is sometimes called the Hurst exponent. For $H=1 / 2$ the classical diffusion result is obtained (Brownian motion), $H>1 / 2$ indicates correlated diffusion, which we expect in our case. 


\section{EXPERIMENTAL DATA}

To record the trajectory of an individual mouse mammary gland epithelial cell over long time periods of up to 72 hours, epithelial cells 25 were seeded sparsely onto a 2D plastic culture dish. The dish was then placed into a mini-chamber mounted on an inverted phase contrast video-microscope. This provided incubator conditions and periodic image acquisition in 90 seconds intervals was performed [26]. About 900 to 1000 (video) frames were taken per measurement. For our purposes we only take the first 25 hours of the recording since after that cell density becomes high, and motion of individual cells restricted, see video (http://is2.kph.tuwien.ac.at/thurner/CELLMOVE). As seen in the videos, cells move within limited regions for many timesteps, crossing and following its own and other cell's trajectories.

From the video sequences the motion of individual cells was extracted semi-automatically by a commercial motion tracking routine [27]. Cell nuclei were tracked based on a convolution kernel of a 100 square pixel field (about half the size of the cell nucleus at the given resolution). These extracted cell trajectories, see lines in Fig. 1b and c, were recorded as the $x_{t}$ and $y_{t}$ positions in two dimensions (the subscript indicating discrete time).

Due to the limited data length it is more useful to study velocity distributions rather than spatial distributions directly. We work in polar coordinates and transform the trajectories $\left(x_{t}, y_{t}\right)$ to a velocity (length-increment $\left.v_{x}(t)=x_{t}-x_{t-1}\right)$ process $v_{t}=\sqrt{v_{x}(t)^{2}+v_{y}(t)^{2}}$ and a direction process $\alpha_{t}=\arctan \left(x_{t}-x_{t-1}, y_{t}-y_{t-1}\right) \in[-\pi, \pi]$.

As a control for the plausibility of our model we genetically modified cells from the same cell line in two virus experiments [28, 29, 30]. The basic idea here is to alter the cell's motion program such that its interaction with other cells and hence its ability to follow other cell trajectories becomes effectively reduced. In the case of non-interacting cells one should expect the model loose its validity, i.e. that cell movement would approach classical diffusion, i.e. $q \longrightarrow 1$. In the first experiment, we infected cells with a virus carrying the coding sequence of enhanced green fluorescent protein (GFP). Since viral infection has been reported to significantly influence morphology and molecular homeostasis of mammary epithelial cells [31, 32, 33, 34, we expected alterations on cellular migration patterns as well. This condition will be referred to as "infected" in the following. In a second experiment we inserted the coding sequence of gene TPA-Inducible Sequence 7 (TIS7) instead of GFP into 
the cells. TIS7, a co-repressor of gene transcription and functionally associated with loss of epithelial characteristics like cell-to-cell adhesion was chosen to additionally test for eventual alterations in cell mobility [29]. This experimental condition will be called "TIS7" in the remainder of the paper and contains consequences of the viral infection itself and the effects of over-expressed TIS7.

\section{RESULTS}

For a first demonstration of correlated motion to be present, we compute $C(\tau)$ for the individual $x_{t}$ and $y_{t}$ components of cell trajectories. We find a Hurst exponent of about $H \sim 0.7 \pm 0.02$ for all the experimental conditions (normal, infected, and TIS7), indicating correlated motion, see Tab. 1.

We find the direction process $\alpha_{t}$ practically equally distributed over the interval $[-\pi, \pi]$. By looking at normalized autocorrelations $A C$ of $\alpha_{t}$, significant (99\% significance level) coefficients are found which decay as a power law. The situation is shown in Fig. Ea for all three experimental conditions. Power decay exponents were fit by a least square routine in the region indicated by the straight lines; the corresponding values are collected in Tab. 1. Errors (standard mean errors over 5 independently moving cells) in the fit-regions are somewhat larger than symbol-size and were omitted for clarity of the plot. Normal cells show lower correlations, which decay slightly faster than those of the manipulated cells.

The normalized autocorrelations for the velocity process $v_{t}$ are given in Fig. 2 $\mathrm{b}$. Correlation coefficients are statistically significant and in the same order of magnitude as the direction series'. Again, the tails of the correlations follow a power law; for the corresponding exponents, see Tab. 1. Here normal cells have higher correlations than infected ones. Infected and TIS7 cells show a slower decay than normals. Errors here are about twice symbol size.

In Fig. 3 we show the velocity distribution (histogram) of the normal cells in a log-log plot. The solid line is a three parameter fit to Eq. 幽; fit-parameters for $q$ and $\frac{\beta m}{2}$ for all conditions are listed in Tab. 1. At the low limit of $v$ the distribution approaches a constant, while the right tail clearly decays in a power law fashion $\sim v^{-\gamma}$. We find infected and TIS7 cells to move faster on average, as seen in the mobility factor or directly in $\left\langle v_{t}\right\rangle$, see Tab. 1. 


\section{DISCUSSION}

We provide clear evidence that cell migration of interacting cells is a correlated diffusion process. This finding is supported by several facts found in our experimental data. Correlated motion is shown by the presence of a Hurst exponent significantly larger than 0.5 and by non-vanishing autocorrelation functions in the velocity and direction processes. This finding is in nice agreement with a recent and similar experiment [7], where an $H$ of $0.62 \pm 0.05$ was found. The direction autocorrelation functions are found to decay in a power like manner, with exponents consistently smaller than 1, opening the possibility for superdiffusion. This obviously excludes naive autoregressive or moving average models (ARMA) to be realistic candidates for interacting cell migration since these models have exponential decays of autocorrelation functions. This finding means that the direction of cells is a "long memory" process. For velocity autocorrelation functions the power decay is smaller but still present for the normal cells. The value of the correlation coefficient at $\tau=1$ is about the same size as for the $\alpha_{t}$ process.

The virus effect is seen in the following way. The most obvious difference of normal and manipulated cells is seen in their mobility, which is drastically enhanced for manipulated cells. This is expected because we believe that there is less effective adhesion proteins present in the infected and especially in the TIS7 cells. Very similar results have been reported in [7], however, with a different experimental strategy: There cells propagated in different aggregates of cell types with different adhesion properties to Hydra cells.

Infected and TIS7 cells show larger correlations in the direction process, meaning that directions are changed less frequently than normal cells do. This is expected since these cells detect trails less well and lose their ability to follow other cells. Once moving they keep their direction more strictly than normal cells. For the velocity process we observe the opposite effect: normal cells have larger autocorrelations, i.e. normal cells change their velocities less frequently than infected and TIS7 ones. We attribute this effect to a more controlled movement of normal cells when following a path, or equally, a stronger influence of random effects on infected or TIS7 cells with damaged movement control.

We now turn to the main result of this paper. The velocity distribution follows the general form of the anomalous diffusion model. Maybe most importantly this model allows to naturally understand the presence of the power tail to the right of the distribution. The 
power can be related (via $q$ ) to the $\nu$ exponent in Eq. (1). For normal cells we find $q \sim 1.35$ which goes down to $q \sim 1.20$ for the infected, and to $q \sim 1.11$ for the TIS7 cells. In the Tsallis thermodynamics framework this means, that infected cells approach the classical thermodynamic regime, while normal cells with their complex motion control system are governed by non-extensive statistics. In this framework it is clear that for non-interacting cells ordinary diffusion and Gaussian or exponential velocity distributions were observed in [21, 22], being extreme cases of the present model. If we accept that in our experiments infection diminishes the degree of interaction between cells and their trajectories, we see that the $q$ value should serve as a sensible quantifier of the degree of interaction. Again, in [7] very similar behavior of 2D moving patterns of Hydra cells were found. They reported $q \sim 1.5$, or a power decay in the velocity distributions of about $\gamma \sim 4$ and suggest a different way of explaining the movement patterns based on granular flow models. Furthermore, these and our findings clearly exclude a Levy flight interpretation of cell movements, and led by the observation of trail-following tendencies of cells we believe that cell migration is governed by a realization of anomalous diffusion.

In conclusion, we think that the presented method provides a useful tool to qualitatively understand different movement patterns of realistic cell migration, and to quantify the degree of interaction by the value of the Tsallis $q$. Since epithelial cell migration plays such an important role in situations like wound healing of skin epidermis or cancer metastasis we speculate that the above way of quantification could even be a practical pathological characterization of cells, regarding diverse biological dignities and pharmacological responses, in addition to molecular and mechanical parameters. Finally, we note that the basic idea behind the presented model could also be applicable to other fields, like electrical flow through networks of biological neurons. There the effective network topology is also subject to constant changes due to synaptic plasticity (Hebb or other type of learning). Electrical current will flow on excitable media restricted to a given network and by doing so, will re-shape it by changing synaptic weights. Here we would predict power law distributions of electrical current changes, in analogy to the $v$ distributions presented here.

S.T. would like to thank C. Tsallis and M. Gell-Mann for the inspiring workshop on non-extensive thermodynamics they organized at the Santa Fe Institute in April 2002. We acknowledge M. Cotten for the preparation of CELO vector stocks. This work was supported by Boehringer Ingelheim and by the Austrian Science Foundation grant FWF, P13577-GEN. 
[1] C. Tsallis, J. Stat. Phys. 52 (1988) 479.

[2] C. Tsallis and D.J. Bukman, Phys. Rev. E 54 (1996) 2197.

[3] C. Tsallis, R.S. Mendes and R. Plastino, Physica A 261 (1998) 534.

[4] A.R. Plastino and A. Plastino, Phys. Lett. A 174 (1993) 384.

[5] G. Kaniadakis, A. Lavagno and P. Quarati, Phys. Lett. B 369 (1996) 308.

[6] M.L. Lyra and C. Tsallis, Phys. Rev. Lett. 80 (1998) 53.

[7] A. Upadhyaha, J.P. Rieu, J.A. Glazier and Y. Sawada, Physica A 293 (2001) 549.

[8] D.A. Lauffenburger and A.F. Horwitz, Cell 84 (1996) 359.

[9] J. Lee, et al., Nature 362 (1993) 167.

[10] L. Hennighausen and G.W. Robinson, Genes Dev. 12 (1998) 449.

[11] G.W. Robinson, et al., Development 121 (1995) 2079.

[12] P. Schedin, T. Mitrenga and M. Kaeck, J. Mammary Gland Biol. Neoplasia 5 (2000) 211.

[13] M.J. Bissell, et al., Cancer Res. 59 (1999) 1757.

[14] J.S. Foster, et al., Trends Endocrinol. Metab. 12 (2001) 320.

[15] C.C. Park, M.J. Bissell and M.H. Barcellos-Hoff, Mol. Med. Today 6 (2000) 324.

[16] J. Aggeler, et al., J. Cell Sci. 99 (1991) 407.

[17] I. Fialka, et al., J. Cell Biol. 132 (1996) 1115.

[18] M. Chicurel, Science 295 (2002) 606.

[19] F. Palacios, L. Price, J. Schweitzer, J.G. Collard and C. D’Souza-Schorey, EMBO J. 20 (2001) 4973.

[20] E. Hintermann, M. Bilban, A. Sharabi and V. Quaranta, J. Cell Biol. 153 (2001) 465.

[21] J.C.M Mombach and J.A. Glazier, Phys. Rev. Lett. 76 (1996) 3032.

[22] A. Czirok, et al., Phys. Rev. Lett. 81 (1998) 3038.

[23] M. Bologna, C. Tsallis and P. Grigolini, Phys. Rev. E 62 (2000) 2213.

[24] R. Silva, A.R. Plastino and J.A.S. Lima, Phys. Lett. A 249 (1998) 401.

[25] Generation of the cell line (c-JunER) has been described in [17]. Media, tissue culture reagents, and FCS (Fetal Calf Serum) were purchased from GIBCO BRL and Boehringer Mannheim. Cells were routinely cultured in High Glucose DMEM supplemented with 5\%FCS, 10mM Hepes, $\mathrm{pH} 7.3$, $50 \mathrm{IU} / \mathrm{ml}$ penicillin and $50 \mathrm{mg} / \mathrm{ml}$ streptomycin. As infection medium High 
Glucose DMEM supplemented with 2\%FCS and 10mM Hepes was used. The generation of the viral vector and the protocol of infection have been described in [28, 29] and have followed except that 9000 viral particles per cell have been used in order to achieve maximum infection efficiency.

[26] Real-time videomicroscopy was done with an inverted phase contrast microscope (Axiovert 35, Carl Zeiss Inc.) equipped with an on-stage minichamber providing routine incubation conditions $\left(37^{\circ} \mathrm{C}, 5 \% \mathrm{CO} 2\right)$. As a system control a replicon was carried along in the incubator used for routine cell culture (data not shown). Phase contrast images were taken in 90 second intervals using a Shutter Driver (UniBLITZ D122) as a frame grapper. (http://is2.kph.tuwien.ac.at/thurner/CELLMOVE).

[27] MetaMorph Imaging System 4.6, Universal Imaging Corporation.

[28] A.I. Michou, et al., J. Virol. 73 (1999) 1399.

[29] V.S. Vietor, et al., EMBO J. XX (2002) XX, in print.

[30] The viral vector derived from the chicken embryo lethal orphan and fowl adenovirus type1 and has been shown to efficiently serve in overexpression of ectopic proteins in epithelial cells [29].

[31] R.B. Gaynor, L.T. Feldman and A.J. Berk, Science 230 (1985) 447.

[32] J.B. Glotzer, et al., Nature 407 (2000) 207.

[33] J.Wolff, et al., Exp. Cell Res. 202 (1992) 67.

[34] N. Wick, et al., (2002) in preparation. 


\begin{tabular}{lccc} 
Measure & normal cells & infected & TIS7 \\
\hline$q$ & 1.350 & 1.201 & 1.110 \\
$\frac{\beta m}{2}$ & 1.20 & 0.70 & 0.30 \\
$\left\langle v_{t}\right\rangle$ & $38.8 \mu \mathrm{m} / \mathrm{h}$ & $51.7 \mu \mathrm{m} / \mathrm{h}$ & $71.7 \mu \mathrm{m} / \mathrm{h}$ \\
$H$ & $0.686(24)$ & $0.700(18)$ & $0.739(31)$ \\
AC decay exponent: $v_{t}$ & -0.095 & 0.026 & -0.009 \\
AC decay exponent: $\alpha_{t}$ & -0.713 & -0.701 & -0.591 \\
\hline
\end{tabular}

TABLE I: Values for the Tsallis $q$, mobility factors $\frac{\beta m}{2}$, and average cell velocities $\left\langle v_{t}\right\rangle$, for the three cell types. Data was obtained by LS fits to the aggregated velocity distributions. Additionally, we show mean and standard error (from averages over individual trajectories) for the Hurst exponent and fits to power decay exponents of the tails in the autocorrelation functions of the cell velocities $v_{t}$ and the direction process $\alpha_{t}$. 
FIG. 1: Normal mouse mammary gland epithelial cells migrating on a two-dimensional culture surface. Large magnification of a single cell (a). The leading edge can be recognized as a fanshaped lamellipodium (small arrows) at the bottom of the panel. The trailing edge is marked by a star. The direction of migration is indicated by the fat arrow. (b) Extracted trajectory of a single normal cell over 1000 timesteps $(25 \mathrm{~h})$.

FIG. 2: Autocorrelation function of the direction process $\alpha_{t}$ of cell migration for normal, infected, and TIS7 cells (a). Infected and TIS7 cells have somewhat higher values in the correlations and show a softer decay. (b) Autocorrelations for the velocity process $v_{t}$. For infected and TIS7 cells the coefficients are lower and the curves are nearly constant, while normal cells again show a power decay.

FIG. 3: Cell velocity distribution (histogram) for normal cells. For small $v$ the distribution approaches a constant and decays for larger $v$ in a clear power law. The line represents a fit to the Tsallis velocity distribution. The virus experiments are not shown for clarity; their fit parameters are gathered, however, in Tab. 1. 

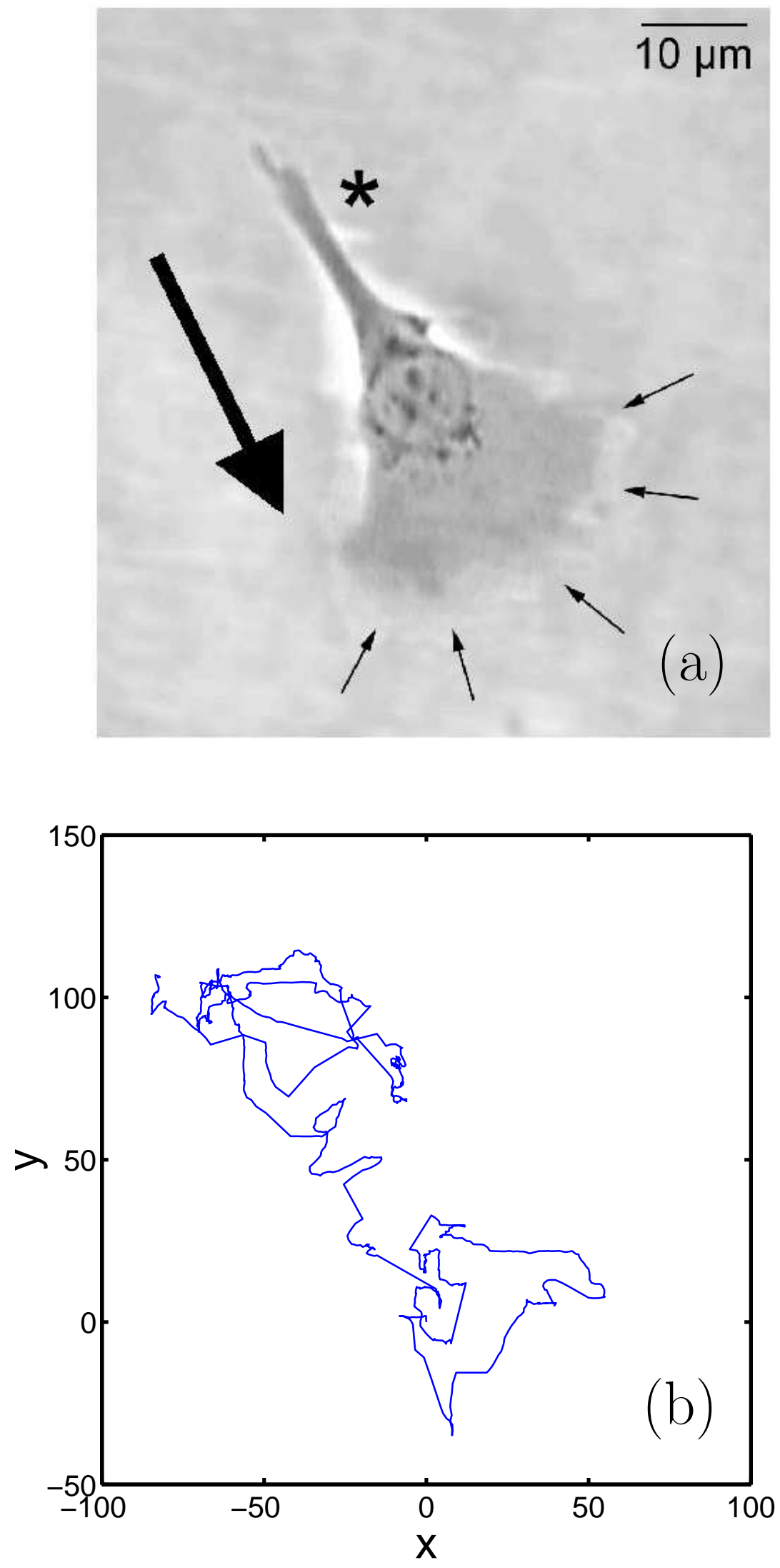

FIG. 1 

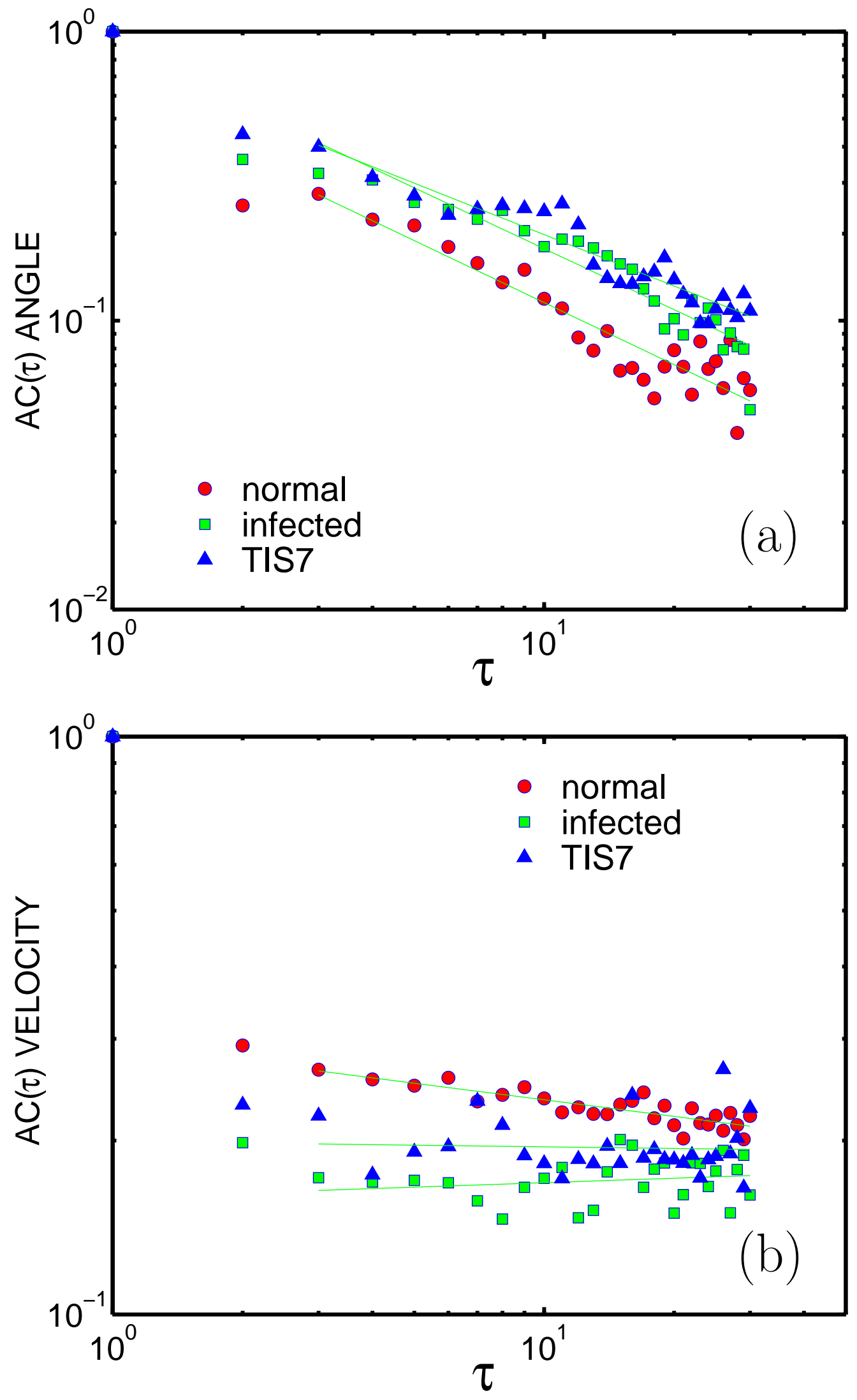

FIG. 2 


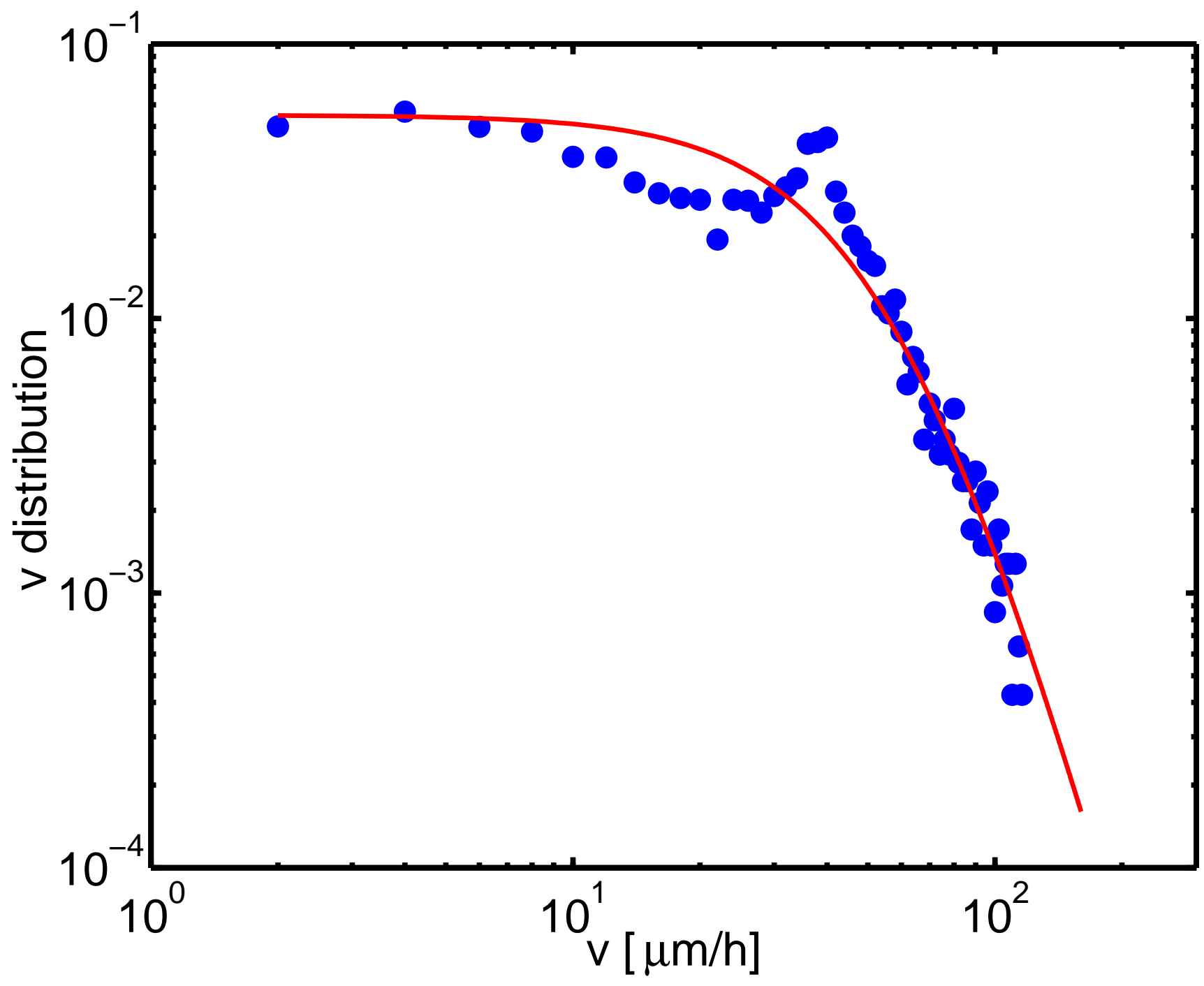

FIG. 3 